\section{A case of relapsing polychondritis mimicking Ludwig's angina}

\author{
Adnan Agha,' AlHussain Asiri, ${ }^{1}$ \\ Ali Shehri, ${ }^{1}$ Abdelhaleem Bella, \\ Mohamed Bazeed ${ }^{2}$
}

'Department of Internal Medicine; 2Department of Radiology, Armed Forces Hospital Southern Region, Khamis Mushyt, Kingdom of Saudi Arabia

\section{Abstract}

Relapsing polychondritis (RP) is a severe progressive inflammatory condition involving cartilaginous structures and caused by an autoimmune process, for which there is no confirmatory serological biomarker and which still is diagnosed on a mainly clinical basis. RP has been associated with many diseases like Sweet's syndrome, Behcet's disease, rheumatoid arthritis, and other autoimmune disorders. We attempt to describe here a unique case of a 38-year-old female with a high-grade fever, sore throat, difficulty in swallowing, hoarseness of the voice, and found to have signs of tongue swelling and inflammation of the oral cavity that mimicked Ludwig's angina, necessitating antibiotic use. On careful reevaluation the patient was diagnosed to have relapsing polychondritis based on auricular and respiratory tract chondritis and response to steroids. The purpose of this report is to emphasize the fact that careful clinical assessment is needed to diagnose RP, which may be misdiagnosed as Ludwig's angina.

\section{Case Report}

A 38-year-old Saudi female, previously not known to have any medical illnesses, presented to the emergency room with complaints of high-grade continuous fever, sore throat, difficulty in swallowing, and hoarseness of the voice for five days prior to admission. Her fever was relieved only partially with antipyretics. In addition she gave a history of painful swallowing both for liquids and solids, which had gradually progressed over the last three days, making her unable to take anything orally. This symptom was associated with hoarseness of the voice. She had no significant past medical history, especially for any recurrent upper respiratory tract infections, joint pains, oral ulcerations, eye symptoms, or fever. On examination by the ENT specialist, she was found to have a swollen tongue that was ulcerated and coated, and the tonsils could not be visualized owing to the pain and swelling of the tongue. There was some tenderness over the neck region, with enlargement of bilateral cervical lymph nodes and tenderness bilaterally over the ears. There was a palpable $5 \times 3 \mathrm{~cm}$ posterior collar mass. The patient's laboratory data were most significant for leukocytosis, as seen in Table 1. Based on the clinical and laboratory data a provisional diagnosis of Ludwig's angina was made and the patient was started on cefuroxime (750 mg IV t.i.d.) and metronidazole (500 mg IV t.i.d.). The blood, sputum, and oral cavity secretions were sent for culture analysis but no organisms grew.

The patient remained febrile and had continued to have stridor. Her spirometry findings showed a respiratory obstructive element with a forced expiratory volume/forced vital capacity (FEV1/FVC) of $66.1 \%$ that was indicative of obstructive airway disease, while her forced expiratory volume/peak expiratory flow ratio (FEV1/PEFR) was 5.8, which pointed towards involvement of the upper airways. Her ambient oxygen saturation was $95 \%$. The chest X-ray was normal as was the CT scan of the thorax, which only showed some bilateral axillary lymph nodes of insignificant size (Figure 1). Fluconazole and valcyclovir were added to her medications, and cefuroxime was changed to amoxicillin/clauvadonic acid. A CT scan of the neck was performed on the third day, which showed a swollen tongue, thickening of the left tonsillar and retropharyngeal tissue superior to the larynx, with multiple enlarged lymph node chains in the neck (Figure 2). After a review by the rheumatologist, and based on
Correspondence: Adnan Agha, Registrar Department of Internal Medicine, Armed Forces Hospital Southern Region, Khamis Mushyt, P.0. Box 101, Kingdom of Saudi Arabia

Email: adnanagha@hotmail.com

Received for publication: 29 May 2009.

Revision received: 24 August 2009.

Accepted for publication: 24 August 2009

This work is licensed under a Creative Commons Attribution 3.0 License (by-nc 3.0)

(C) Copyright A. Agha et al., 2009

Licensee PAGEPress, Italy

Rheumatology Reports 2009; 1:e6

doi:10.4081/rr.2009.e6

her history of recurrent inflammation of the nasal and tracheal cartilages, with current clinical evidence of respiratory tract chondritis and nasal chondritis characterized by tenderness over these areas, a diagnosis of relapsing polychondritis (RP) was made. No abnormality was detected on ophthalmological examination and there was no history of arthralgias, arthritis or any skin or genital rashes, pigmentation or ulcerations, nor a family history of autoimmune disease or cancer. Her laboratory data were not diagnostic for other related autoimmune diseases or hypothyroidism. The patient was treated with steroids in the form of oral prednisone (1 mg/kg/day) after a three-day pulse of methylprednisone (250 mg IV daily) together with supportive IV hydration. Calcium and vitamin D supplements were given to her with methotrexate (10 mg per mouth) once a

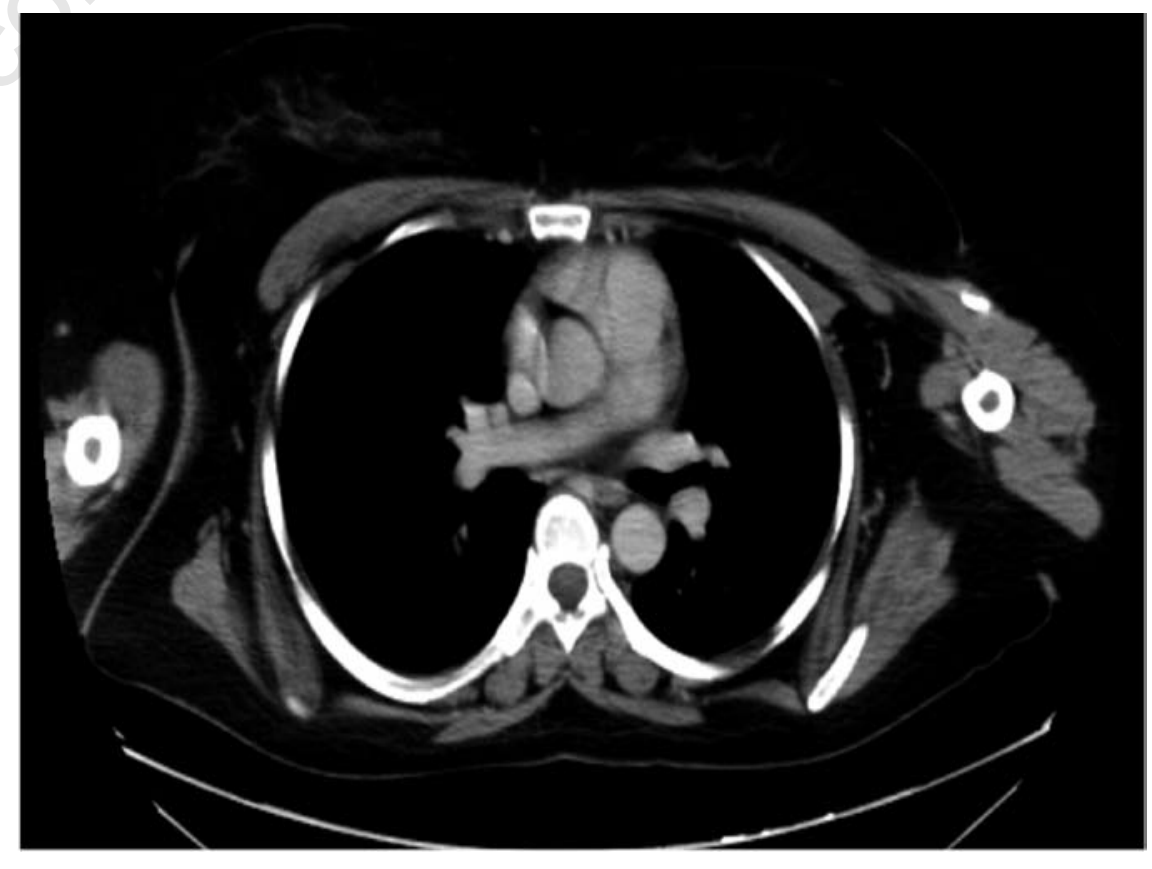

Figure 1. Computed tomography scan of the thorax showing no significant abnormality in the lungs. 
week. The patient showed a dramatic response and within five days she was completely asymptomatic. She had obstructive, nonreversible airway disease on presentation (FEV1/FVC ratio of 66\%), which had improved on treatment (ratio becoming $76.6 \%$ ), while the FEV1/PEFR ratio progressed from 5.8 to 5.2 suggesting improvement in the upper airway obstruction. She was discharged home on the seventh day on a tapering dose of steroids (prednisone, $30 \mathrm{mg}$ per day decreasing by $5 \mathrm{mg}$ per week) and methotrexate, although some mild tracheal cartilage tenderness and mild pharyngeal erythema were present still. On a follow-up visit after six weeks the patient had total resolution of symptoms with no evidence of any inflammation. The methotrexate was increased to $15 \mathrm{mg}$ per week and the prednisone was reduced to $5 \mathrm{mg}$ per day, to be discontinued after one week.

\section{Discussion}

RP is a severe, episodic, and progressive inflammatory condition involving cartilaginous structures, predominantly the ears, nose, and laryngotracheobronchial tree. It is caused by an autoimmune process characterized by infiltrating T-cells, the presence of antigenantibody complexes in affected cartilage, cellular and humoral responses against collagen type II and other collagen antigens. ${ }^{1}$ The McAdam's Diagnostic Criteria for Relapsing Polychondritis stipulates that three or more of the clinical signs must be present (Table 1), while modified criteria include one or more signs with histological confirmation, or chondritis in two or more separate sites and a response to steroids or immunosuppression (Table 2). ${ }^{2}$ Other aids to diagnosing relapsing polychondritis apart from the clinical criteria and histopathology include testing for antibodies against cartilage; unfortunately this expensive laboratory facility was not available in our hospital.

Common signs and symptoms consist of auricular chondritis (present in $85-95 \%$ of patients); nonerosive seronegative, usually asymmetric, nondeforming, inflammatory polyarthritis (in $52-85 \%$ of patients); nasal chondritis (48-72\% of patients); ocular inflammation (50-65\% of patients) with episcleritis, scleritis, iritis, or retinopathy; respiratory tract chondritis (40-56\% of patients); audiovestibular damage (46-50\% of patients), and cardiovascular disease (24\% of patients) like heart blocks, pericarditis, and valvular and aortic inflammation. ${ }^{3}$ Other signs and symptoms include peripheral ulcerative keratitis, skin lesions like aphthous ulcers, superficial phlebitis, livedo reticularis, limb ulceration with distal necrosis, erythema elevatum
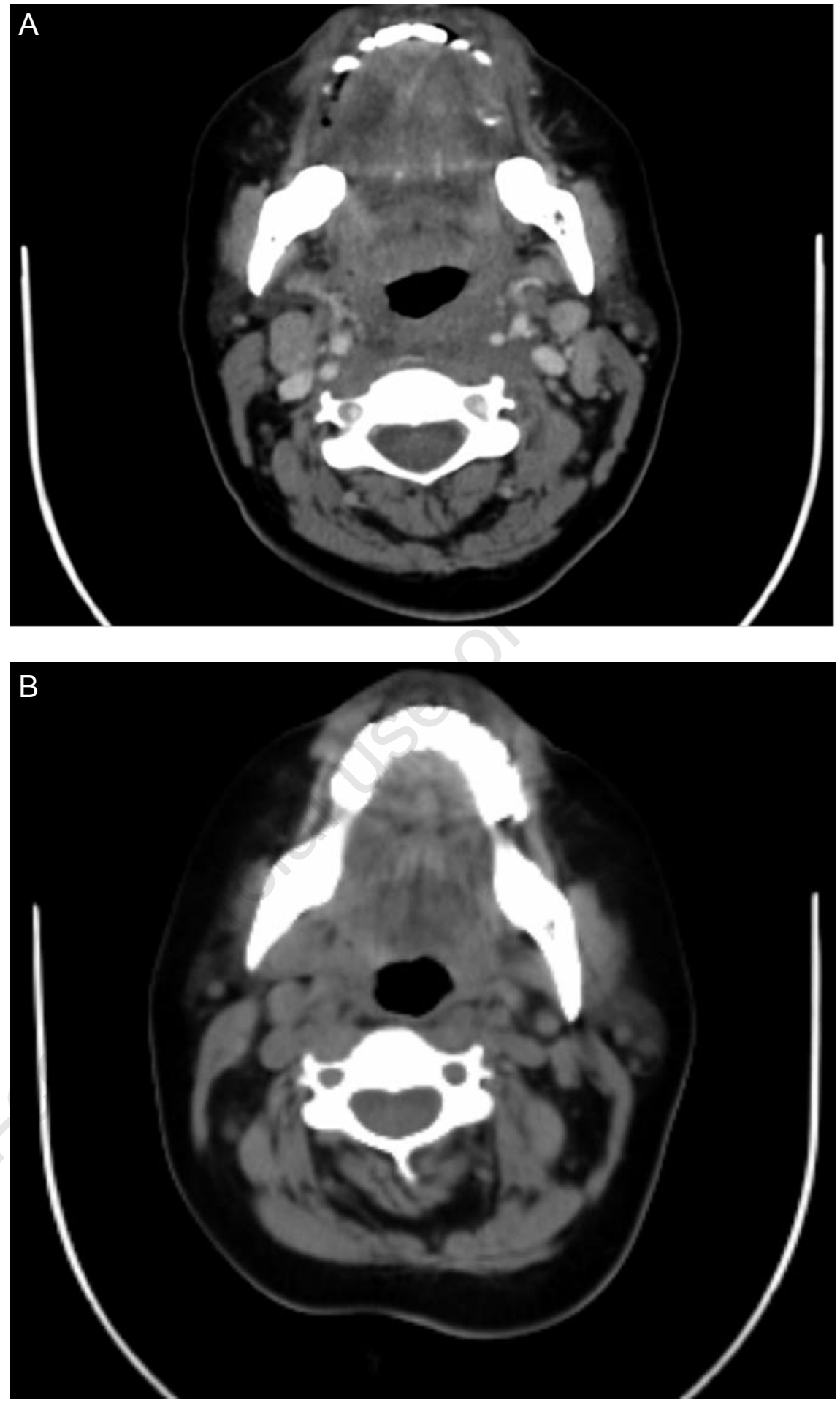

Figure 2. (A) a case of relapsing polychondritis mimicking Ludwig's angina; (B) below, after treatment with steroids, showing improvement.

diutinum, Sweet's syndrome with urticarial vasculitis, leukoclastic vasculitis, and Kaposi sarcoma; panniculitis; central nervous system vasculitis, and aseptic meningitis; and renal involvement in the form of segmental necrotizing glomerulonephritis or renal vasculitis. ${ }^{4-7}$

As far as the respiratory system is concerned, involvement of the cartilaginous struc- tures of the respiratory tract is uncommon at presentation but occurs in almost half of relapsing polychondritis patients during the course of their illness. ${ }^{6}$ Respiratory involvement is a poor prognostic sign and accounts for around $50 \%$ of relapsing polychondritisrelated deaths. Progression of tracheal disease leads to fibrotic changes and diffuse narrowing 
Table 1. Diagnostic criteria for relapsing polychondritis, as suggested by McAdam/ Michet with modification by Damiani-Levine. ${ }^{17,18}$

Criteria

Bilateral auricular chondritis

Nonerosive seronegative inflammatory polyarthritis

Nasal chondritis

Ocular inflammation (conjunctivitis, keratitis, scleritis/episcleritis, uveitis)

Respiratory tract chondritis (of laryngeal and/or tracheal cartilages)

Cochlear and/or vestibular dysfunction (neurosensory hearing loss, tinnitus and/or vertigo)

To establish the diagnosis, all patients were required to have one of the following

At least three of the above clinical criteria.

One or more of the above clinical criteria with positive histological confirmation.

Chondritis at two or more separate anatomic locations, with response to steroids and/or dapsone.

Table 2. Summary of laboratory investigations.

\begin{tabular}{lccc} 
Laboratory data & $\begin{array}{c}\text { On the day } \\
\text { of admission }\end{array}$ & $\begin{array}{c}\text { After 5 days } \\
\text { hospital stay }\end{array}$ & $\begin{array}{c}\text { On discharge } \\
\text { (after 9 days) }\end{array}$ \\
Sodium mmol/L & 138 & 132 & 138 \\
Potassium $\mathrm{mmol} / \mathrm{L}$ & 3.9 & 4.1 & 4.0 \\
\hline Creatinine umol/L & 96 & 61 & 55 \\
Urea $\mathrm{mmol} / \mathrm{L}$ & 5.6 & 2.8 & 5.8 \\
\hline WBC $109 / \mathrm{uL}$ & 14.7 & 14.5 & 11.0 \\
Alanine aminotransferase & 44 & 43 & 45 \\
\hline Bilirubin umol/L & 9 & 10 & 5 \\
\hline
\end{tabular}

\section{Other laboratory workup}

\begin{tabular}{lccc} 
C-reactive protein mg/L & 51.1 & Urine complete & Normal \\
Rheumatoid factor & -ve & Antinuclear antibody & -ve \\
\hline HBsAg & -ve & Anti HCV & -ve
\end{tabular}

Table 3. Summary of pulmonary function tests.

\begin{tabular}{lcc} 
PFI & Jan 2009 & June 2009 \\
Peak expiratory flow (PEF) & $400 \mathrm{~mL}$ & $370 \mathrm{~mL}$ \\
Forced vital capacity (FVC) & $2.74 \mathrm{~L}$ & $2.58 \mathrm{~L}$ \\
\hline Forced expiratory volume per sec (FEV1) & $2.10 \mathrm{~mL} / \mathrm{min}$ & $2.05 \mathrm{~mL} / \mathrm{min}$ \\
FEV1/FVC ratio & $76.6 \%$ & $79.4 \%$ \\
\hline FEV1/PEF ratio & $5.2 \mathrm{~mL} / \mathrm{L} / \mathrm{min}$ & $5.8 \mathrm{ml} / \mathrm{L} / \mathrm{min}$ \\
\hline
\end{tabular}

of the main airway and severe airflow impairment. Persistent bronchial cartilage inflammation may cause impairment of mucociliary function which, along with the use of steroids and other immunosuppressive drugs, predisposes the patient to recurrent respiratory tract infections. Most patients with respiratory involvement assume a fluctuating yet progressive course, and multiple bouts of inflammation eventually lead to permanent destruction of the large airways. In severe cases persistent inflammation can cause acute airway narrowing and destroy the cartilaginous rings, which leads to luminal collapse. Obstruction may be induced iatrogenically by bronchoscopy or tracheostomy. Intubation may be difficult and dangerous because of a small glottis caused by edema or cartilage destruction. One of the rare manifestations of respiratory polychondritis is the involvement of costosternal cartilages, which may lead to costochondritis that impairs breathing. ${ }^{7}$ In addition, our patient had an upper airway obstruction as indicated by the FEV1/PEF ratio. This ratio, in an upper airway obstruction where the insult is in the precarina region, has been shown to be significantly higher than in patients with asthma, chronic obstructive pulmonary disease, or in normal subjects, as the PEF would be affected more than the FEV1 in precarina upper airway obstruction, with the former reflecting more the proximal airway with the FEV1/PEFR value becoming 6 or greater. ${ }^{8}$ Although flexible bronchoscopy is required to confirm this, in our patient the FEV1/PEF ratio improved from 5.8 to 5.2 (Table 3). Our patient also had obstructive disease (FEV1/PEF ratio 66\%) which improved on treatment (FEV1/PEF ratio, 76.6\%, and absolute value improvement in FEV1 of $300 \mathrm{~mL}$ ). The differential diagnoses that need to be ruled out in such patients include Addison's disease, rheumatoid arthritis, Behcet's disease, syphilis, cellulitis of the oral cavity, systemic lupus erythematosus, hyperthyroidism, Wegener's granulomatosis, polyarteritis nodosa, etc. ${ }^{9}$ There are no specific diagnostic tests but markers for inflammation; for example, increased leukocyte count, raised CRP or ESR, an ECG and echocardiogram may show large vessel vasculitis, while MRI and CT scans may show cartilaginous involvement. ${ }^{10}$ The histology of involved cartilage demonstrates chondrolysis, chondritis, and perichondritis. The cartilage loses its basophilia, probably by the release of sulfated proteoglycans from the matrix, and the chondrocytes are decreased in number and may appear pyknotic. ${ }^{11}$ The airway complications in relapsing polychondritis pose a serious diagnostic and therapeutic challenge, and may lead to death as a result of asphyxia. ${ }^{12}$ Cases of RP have been reported in children as well as in adults, with ages ranging from two to 84 years (median, 50 yr) at the time of diagnosis, and the male-tofemale ratio is 1:1; however, among the patients with serious airway manifestations, females predominate (female-to-male ratio, 2.6:1). ${ }^{13}$ The mainstay of treatment is systemic corticosteroids like prednisone $(20-60 \mathrm{mg} / \mathrm{d})$, which decrease the severity, frequency, and duration of relapses of RP. Other medications reported to control progression of the disease include dapsone (25-200 mg/d), azathioprine, methotrexate (7.5-22.5 mg/wk), cyclophosphamide, and cyclosporine. Some case reports have described successful treatment with infliximab and etanercept, antitumor necrosis factor- $\alpha$ antibodies, and anakinra, an interleukin 1 receptor antagonist. ${ }^{6}$

The academically stimulating aspect of this case is the presence of glossitis and oropharyngeal erythema and edema that mimicks Ludwig's angina. Tongue swelling has been reported once before to the best of the authors' knowledge, in a case in which a female with recurrent tongue and facial swelling was thought initially to have angioneurotic edema but was diagnosed later in her life to have relapsing polychondritis. ${ }^{14}$ Another possible explanation for the glossitis may be MAGIC syndrome which is a combination of relapsing polychondritis and Behcet's disease..$^{15}$ However, our patient did not have the other features of Behcet's disease. Therefore, tongue swelling may have been part of severe chondritis of oropharyngeal cartilaginous structures, which is rare in relapsing polychondritis. 


\section{Conclusion}

In the past RP has been associated with many diseases like Sweet's syndrome, which is acute febrile neutrophilic dermatosis, and myelodysplasia. ${ }^{16,17}$ It has been related to other autoimmune diseases like Behcet's syndrome, rheumatoid arthritis, Crohn's disease, systemis lupus erythematosis, and systemic vasculitis. ${ }^{18-20}$ In this case review we attempt to describe a patient with signs and symptoms mimicking cellulitis of the floor of the mouth and tongue (Ludwig's angina), and to stress the fact that RP may be misdiagnosed as such, still being based on clinical grounds almost a century after its discovery. Therefore careful assessment and a high level of vigilance are needed to detect this rare yet classical disease.

\section{References}

1. Letko E, Zafirakis P, Baltatzis S, et al. Relapsing polychondritis: a clinical review. Semin Arthritis Rheum 2002;31:384-95.

2. Molina JF and Espinoza LR. Relapsing polychondritis. Baillieres Best Pract Res Clin Rheumatol 2000;14:97-109.

3. Messmer EM and Foster CS. Vasculitic peripheral ulcerative keratitis. Surv
Ophthalmol 1999;43:379-96.

4. Manghani MK, Andrews J, Higgens CS. Kaposi's sarcoma in a patient with severe relapsing polychondritis. Rheumatol Int 2004;24:309-11.

5. Serratrice J, de Roux-Serratrice C, Ené N, et al. Urticarial vasculitis revealing relapsing polychondritis. Eur J Intern Med 2005; 16:207-8

6. Stewart SS, Ashizawa T, Dudley AW Jr, et al. Cerebral vasculitis in relapsing polychondritis. Neurology 1988;38:150-2.

7. Chang-Miller A, Okamura M, Torres VE, et al. Renal involvement in relapsing polychondritis. Medicine (Baltimore) 1987;66:202-17.

8. Mellisant CF, Van Noord JA, Van de Woestijne KP, et al. Comparison of dynamic lung function indices during forced and quiet breathing in upper airway obstruction, asthma, and emphysema. Chest 1990; 98:77-83.

9. Tesche S, Wenzel S, Sagowski C. Relapsing polychondritis: a case report and review of the literature. Laryngorhinootologie 2005; 84:352-6.

10. Miyasaka LS, de Andrade Júnior A, Bueno $\mathrm{CE}$, et al. Relapsing polychondritis. Sao Paulo Med J 1998;116:1637-42.

11. Wang T, Lu Q, Qin Y, et al. The diagnosis and treatment of relapsing polychondritis (a case report) Lin Chung Er Bi Yan Hou Tou Jing Wai Ke Za Zhi 2008;22:590-2.
12. McAdam LP, O'Hanlan MA, Bluestone R, et al. Relapsing polychondritis. Medicine 1976;55:193-215.

13. Eng J and Sabanathan S. Airway complications in relapsing polychondritis. Ann Thorac Surg 1991;51:686-92.

14. Orme RL, Nordlund JJ, Barich L, et al. The MAGIC syndrome (mouth and genital ulcers with inflamed cartilage). Arch Dermatol 1990;126:940-4.

15. Wilson GE, Hasleton PS, Manns JJ, et al. Relapsing polychondritis: bone marrow and circular fibrous nodules in the aorta. Ann Rheum Dis 1990;49:795-7.

16. Mihulka K. Relapsing polychondritis--a challenge for modern diagnostics and treatment. Przegl Lek 2008;65:77-81.

17. Cohen PR. Sweet's syndrome and relapsing polychondritis: is their appearance in the same patient a coincidental occurrence or a bona fide association of these conditions? Int J Dermatol 2004;43:772-7.

18. Myers B, Gould J, Dolan G. Relapsing polychondritis and myelodysplasia: a report of two cases and review of the current literature. Clin Lab Haematol 2000;22:45-8.

19. Touma DJ. Relapsing polychondritis in association with Crohn's disease. Am J Otolaryngol 1996;17:424-6.

20. Michet CJ Jr, McKenna CH, Luthra HS, et al. Relapsing polychondritis. Survival and predictive role of early disease manifestations. Ann Intern Med 1986;104:74-8. 